\title{
Impact of the Amount of RF Self-interference Cancellation on Digital Self-interference Cancellation in Full Duplex Communications
}

\author{
Si LI, Hong-tao LU, Shi-hai SHAO and You-xi TANG \\ National Key Laboratory of Science and Technology on Communications, University of \\ Electronic Science and Technology of China, Chengdu, China
}

Keywords: Full-duplex (FD), Digital self-interference cancellation.

\begin{abstract}
The self-interference to noise ratio in digital domain decreases as the amount of RF self-interference cancellation increases in full-duplex (FD) communications. The amount of digital self-interference cancellation decreases as the self-interference power in digital domain decreases. The impact of the amount of RF self-interference cancellation on digital self-interference cancellation is analyzed in full-duplex communications. The digital self-interference cancellation analyzed in this paper is based on LS channel estimation. It is shown that the decrease in the amount of digital self-interference cancellation is always less than the increase in RF self-interference cancellation when applying digital cancellation after RF cancellation in full-duplex communications. Applying digital cancellation after RF cancellation is more effective when RF cancellation delivers poor suppression. The performance of digital self-interference cancellation will degrade when RF cancellation achieves large suppression.
\end{abstract}

\section{Introduction}

Full-duplex (FD) transceiver, which transmits and receives simultaneously at the same frequency band, has the potential to double throughput compared with the conventional time division duplex (TDD) and frequency division duplex (FDD) [1-6]. FD transceiver simultaneously receives the desired signal transmitted from the far-end as well as the self-interference coupled from itself. The self-interference is usually of several orders of magnitude higher than the desired signal because the latter crosses much longer distance than the former. Therefore, the fundamental issue of FD transceiver is the mitigation of the self-interference. The existing self-interference cancellation (SIC) mechanisms include antenna cancellation, RF cancellation, and digital cancellation [7-12].

The amount of digital self-interference cancellation when applied after RF self-interference cancellation decreases as the self-interference suppression achieved by $\mathrm{RF}$ cancellation increases when the total self-interference received power is fixed. This leads to the natural question regarding how to allocate the amount of RF and digital self-interference cancellation to make the total amount of self-interference cancellation maximum. The experimental results in [13] show that the effectiveness of digital self-interference cancellation decreases with the increase in the amount of RF self-interference cancellation and digital cancellation is more effective when applied selectively based on measured values. It should be noted that the impact of the amount of RF self-interference cancellation on digital self-interference cancellation in full-duplex communications has not been analyzed in theory yet. 
The expression of digital self-interference cancellation capability and the self-interference received power in digital domain is derived in this paper. The impact of the amount of RF self-interference cancellation on digital self-interference cancellation capability is also analyzed and verified by simulation. Our results show that the decrease in the amount of digital self-interference cancellation is less than the increase in RF self-interference cancellation when RF cancellation achieves large suppression and the decrease in the amount of digital self-interference cancellation is nearly the same as the increase in RF cancellation when RF cancellation delivers small suppression. The digital cancellation is more effective when applied after small RF cancellation.

This rest of the paper is organized as follows. First we present the model of the considered FD system. Then the impact of the amount of RF self-interference cancellation on digital self-interference cancellation capability is analyzed and simulation results are given. Conclusion is presented at the end.

\section{System Model}

Fig. 1 shows the block diagram for the full-duplex node architecture we consider. The near-end receiver receives the desired signal from the far-end transmitter together with the self-interference signal coupled from its own transmitter. We use RF cancellation to reduce the direct path and strong multi-path component from the transmit antenna to receive antenna of the near-end transceiver. Then digital cancellation is applied after RF cancellation to reduce the residual self-interference.

We consider an OFDM architecture employing subcarriers for the transmission. After RF self-interference cancellation, analog to digital converters (ADC) and fast Fourier transform (FFT), the received signal is found to be

$$
\boldsymbol{Y}=\boldsymbol{X}_{\mathrm{D}} \boldsymbol{H}_{\mathrm{D}}+\boldsymbol{X}_{\mathrm{I}} \boldsymbol{H}_{\mathrm{I}}+W
$$

Where $X_{\mathrm{D}}$ and $X_{\mathrm{I}}$ denote the desired signal and self-interference respectively. $\boldsymbol{H}_{\mathrm{D}}$ and $H_{\mathrm{I}}$ denote the frequency response of the desired signal channel and self-interference channel respectively. $\boldsymbol{W}$ is the noise with variance $\sigma^{2}$.

It should be noted that $X_{\mathrm{D}}$ and $X_{\mathrm{I}}$ are diagonal matrices of order $K$ with entries $\left[\boldsymbol{X}_{\mathrm{D}}\right]_{k, k}=X_{\mathrm{D}}(k),\left[\boldsymbol{X}_{\mathrm{I}}\right]_{k, k}=X_{\mathrm{I}}(k) \cdot \boldsymbol{H}_{\mathrm{D}}$ and $\boldsymbol{H}_{\mathrm{I}}$ are column vectors with dimensions $K, \boldsymbol{H}_{\mathrm{D}}=\left[\begin{array}{llll}H_{\mathrm{D}}(0) & H_{\mathrm{D}}(1) & \cdots & H_{\mathrm{D}}(K-1)\end{array}\right]^{\mathrm{T}}$. Where $H_{\mathrm{D}}(k)$ is the frequency response at the $k^{\text {th }}$ tone corresponding to the desired signal channel

$$
H_{\mathrm{D}}(k)=\sum_{l=0}^{L^{\prime}-1} h_{\mathrm{D}}(l) \mathrm{e}^{-j 2 \pi k l / K}
$$

Where $L^{\prime}$ denotes the number of multi-path of the desired signal channel.

$$
\boldsymbol{H}_{\mathrm{I}}=\left[\begin{array}{llll}
H_{\mathrm{I}}(0) & H_{\mathrm{I}}(1) & \cdots & H_{\mathrm{I}}(K-1)
\end{array}\right]^{\mathrm{T}} . \text { Where } H_{\mathrm{I}}(k) \text { is the frequency response at the } k^{\text {th }}
$$
tone corresponding to the self-interference channel

$$
H_{\mathrm{I}}(k)=\sum_{l=0}^{L-1} h_{\mathrm{I}}(l) \mathrm{e}^{-j 2 \pi k l / K}
$$

Where $L$ denotes the number of multi-path of the self-interference channel. 


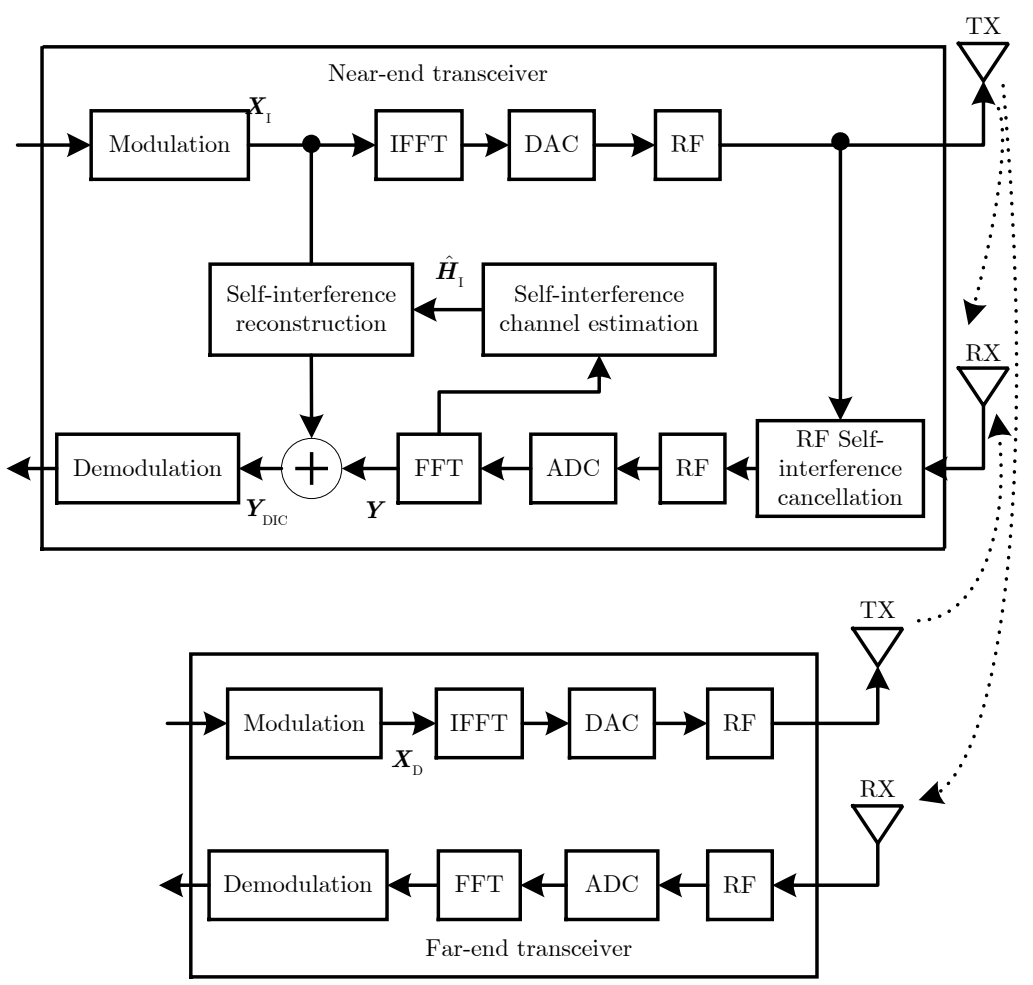

Fig.1. Block diagram of Full-duplex transceiver

As seen in Fig.1, in order to cancel the self-interference in the digital domain, the estimation value of the self-interference channel is first obtained by channel estimation algorithm. Then the self-interference is reconstructed in the digital domain and subtracted from the received signal. The signal after digital cancellation can be expressed as

$$
\boldsymbol{Y}_{\mathrm{DIC}}=\boldsymbol{X}_{\mathrm{D}} \boldsymbol{H}_{\mathrm{D}}+\boldsymbol{X}_{\mathrm{I}}\left(\boldsymbol{H}_{\mathrm{I}}-\hat{\boldsymbol{H}}_{\mathrm{I}}\right)+\boldsymbol{W}
$$

\section{Performance Analysis}

From (4), it can be seen that the estimation value of the self-interference channel is a key factor affecting the performance of the digital self-interference cancellation. Pilot-aided channel-estimation scheme is employed in this paper. The frequency response of the self-interference channel at pilot locations is denoted by $\boldsymbol{H}_{\mathrm{I}}^{(p)}, N_{p}$ is the number of the pilots. Denoting by $\boldsymbol{Y}^{(p)}$ the $N_{p}$-dimensional vector containing the FFT output at the pilot locations, we have

$$
\boldsymbol{Y}^{(p)}=\boldsymbol{X}_{\mathrm{I}}^{(p)} \boldsymbol{H}_{\mathrm{I}}^{(p)}+\boldsymbol{X}_{\mathrm{D}}^{(p)} \boldsymbol{H}_{\mathrm{D}}^{(p)}+\boldsymbol{W}
$$

Where $\boldsymbol{X}_{\mathrm{I}}^{(p)}$ and $\boldsymbol{X}_{\mathrm{D}}^{(p)}$ are diagonal matrices of order $N_{p}$ with entries $\left[\boldsymbol{X}_{\mathrm{I}}^{(p)}\right]_{k, k}=X_{I}\left(i_{k}\right),\left[\boldsymbol{X}_{\mathrm{D}}^{(p)}\right]_{k, k}=X_{\mathrm{D}}\left(i_{k}\right),\left\{i_{n} ; 1 \leq n \leq N_{p}\right\}$ denotes the locations of the pilots. $\boldsymbol{H}_{\mathrm{I}}^{(p)}=\left[\begin{array}{llll}H_{\mathrm{I}}\left(i_{1}\right) & H_{\mathrm{I}}\left(i_{2}\right) & \cdots & H_{\mathrm{I}}\left(i_{N_{p}}\right)\end{array}\right]^{\mathrm{T}}, \boldsymbol{H}_{\mathrm{D}}^{(p)}=\left[\begin{array}{llll}H_{\mathrm{D}}\left(i_{1}\right) & H_{\mathrm{D}}\left(i_{2}\right) & \cdots & H_{\mathrm{D}}\left(i_{N_{p}}\right)\end{array}\right]^{\mathrm{T}}$. 
Define $\boldsymbol{F}=\left[\begin{array}{cccc}1 & W_{K}^{i_{1}} & \cdots & W_{K}^{i_{1}(L-1)} \\ 1 & W_{K}^{i_{2}} & \cdots & W_{K}^{i_{2}(L-1)} \\ \vdots & \vdots & \ddots & \vdots \\ 1 & W_{K}^{i_{N_{p}}} & \cdots & W_{K}^{i_{N_{p}}(L-1)}\end{array}\right], \boldsymbol{F}^{\prime}=\left[\begin{array}{cccc}1 & W_{K}^{i_{1}} & \cdots & W_{K}^{i_{1}\left(L^{\prime}-1\right)} \\ 1 & W_{K}^{i_{2}} & \cdots & W_{K}^{i_{2}\left(L^{\prime}-1\right)} \\ \vdots & \vdots & \ddots & \vdots \\ 1 & W_{K}^{i_{N_{p}}} & \cdots & W_{K}^{i_{N_{p}}}\left(L^{\prime}-1\right)\end{array}\right]$

where $W_{K}=\mathrm{e}^{-j 2 \pi / K}$, then $\boldsymbol{H}_{\mathrm{I}}^{(p)}=\boldsymbol{F}_{\mathrm{I}}, \boldsymbol{H}_{\mathrm{D}}^{(p)}=\boldsymbol{F}^{\prime} \boldsymbol{h}_{\mathrm{D}},(5)$ can be written as

$$
\boldsymbol{Y}^{(p)}=\boldsymbol{X}_{\mathrm{I}}^{(p)} \boldsymbol{F} \boldsymbol{h}_{\mathrm{I}}+\boldsymbol{X}_{\mathrm{D}}^{(p)} \boldsymbol{F}^{\prime} \boldsymbol{h}_{\mathrm{D}}+\boldsymbol{W}
$$

Without loss of generality, most of the self-interference from the direct path is cancelled after RF cancellation. The residual self-interference is mainly from the scattering multipath. Assuming the digital self-interference channel vector $h_{\mathrm{I}}$ is Rayleigh distributed and the entries of $\boldsymbol{h}_{\mathrm{I}}$ are zero-mean independent Gaussian random variables with variance $\sigma_{k}^{2}, k=0,1, \cdots L-1$.

We estimate the vector $h_{\mathrm{I}}$ by the least square (LS) method. From (6), we obtain

$$
\begin{aligned}
\hat{\boldsymbol{h}}_{\mathrm{I}, \mathrm{LS}} & =\left(\left(\boldsymbol{X}_{\mathrm{I}}^{(p)} \boldsymbol{F}\right)^{\mathrm{H}} \boldsymbol{X}_{\mathrm{I}}^{(p)} \boldsymbol{F}\right)^{-1}\left(\boldsymbol{X}_{\mathrm{I}}^{(p)} \boldsymbol{F}\right)^{\mathrm{H}} \boldsymbol{Y}^{(p)} \\
& =\boldsymbol{h}_{\mathrm{I}}+\left(\left(\boldsymbol{X}_{\mathrm{I}}^{(p)} \boldsymbol{F}\right)^{\mathrm{H}} \boldsymbol{X}_{\mathrm{I}}^{(p)} \boldsymbol{F}\right)^{-1}\left(\boldsymbol{X}_{\mathrm{I}}^{(p)} \boldsymbol{F}\right)^{\mathrm{H}} \boldsymbol{X}_{\mathrm{D}}^{(p)} \boldsymbol{F}^{\prime} \boldsymbol{h}_{\mathrm{D}} \\
& +\left(\left(\boldsymbol{X}_{\mathrm{I}}^{(p)} \boldsymbol{F}\right)^{\mathrm{H}} \boldsymbol{X}_{\mathrm{I}}^{(p)} \boldsymbol{F}\right)^{-1}\left(\boldsymbol{X}_{\mathrm{I}}^{(p)} \boldsymbol{F}\right)^{\mathrm{H}} \boldsymbol{W}
\end{aligned}
$$

Orthogonal pilot tones design in [14] is used to reduce the mean square error of the self-interference channel estimate based on LS algorithm. The second item in (7) will be equal to zero when orthogonal pilot tone design is used. Then, (7) can be expressed as:

$$
\hat{\boldsymbol{h}}_{\mathrm{I}, \mathrm{LS}}=\boldsymbol{h}_{\mathrm{I}}+\left(\left(\boldsymbol{X}_{\mathrm{I}}^{(p)} \boldsymbol{F}\right)^{\mathrm{H}} \boldsymbol{X}_{\mathrm{I}}^{(p)} \boldsymbol{F}\right)^{-1}\left(\boldsymbol{X}_{\mathrm{I}}^{(p)} \boldsymbol{F}\right)^{\mathrm{H}} \boldsymbol{W}
$$

Orthogonal pilot tones design schemes include frequency-division multiplexing pilot allocation, time-division multiplexing pilot allocation, and code-division multiplexing pilot allocation[15]. In this paper, frequency-division multiplexing pilot allocation is adopted. In order to analyze the relation between the RF self-interference cancellation capability and the digital self-interference cancellation capability, the mean square error of the LS self-interference channel estimate should be reduced as much as possible to improve the digital self-interference cancellation capability. The mean square error of the self-interference channel estimate in the digital domain can be expressed as

$$
\gamma \triangleq \frac{1}{K} \mathrm{E}\left[\left|\hat{\boldsymbol{H}}_{\mathrm{I}}-\boldsymbol{H}_{\mathrm{I}}\right|^{2}\right]
$$

The mean square error of the LS self-interference channel estimate in the digital domain can be minimized by setting the near-end self-interference pilot tones being equipowered and equispaced ${ }^{[16]}$,i.e., $i_{m}-i_{m-1}=K / N_{p}, \mathrm{E}\left[\left|X_{\mathrm{I}}\left(i_{m}\right)\right|^{2}\right]=1, m=1,2, \cdots N_{p}$. 
From (9), the mean square error of the LS self-interference channel estimate can be written as

$$
\begin{aligned}
\gamma_{\mathrm{LS}} & =\frac{1}{K} \mathrm{E}\left[\left|\boldsymbol{F}\left(\hat{\boldsymbol{h}}_{\mathrm{I}, L S}-\boldsymbol{h}_{\mathrm{I}}\right)\right|^{2}\right] \\
& =\frac{1}{K} \mathrm{E}\left[\left.\boldsymbol{F}\left(\left(\boldsymbol{X}_{\mathrm{I}}^{(p)} \boldsymbol{F}\right)^{\mathrm{H}} \boldsymbol{X}_{\mathrm{I}}^{(p)} \boldsymbol{F}\right)^{-1}\left(\boldsymbol{X}_{\mathrm{I}}^{(p)} \boldsymbol{F}\right)^{\mathrm{H}} \boldsymbol{W}\right|^{2}\right]
\end{aligned}
$$

Assuming $\boldsymbol{A}_{\mathrm{I}}=\boldsymbol{X}_{\mathrm{I}}^{(p)} \boldsymbol{F}, \boldsymbol{A}_{\mathrm{I}}^{+}=\left(\boldsymbol{A}_{\mathrm{I}}^{\mathrm{H}} \boldsymbol{A}_{\mathrm{I}}\right)^{-1} \boldsymbol{A}_{\mathrm{I}}^{\mathrm{H}}$, then (10) can be written as

$$
\begin{aligned}
\gamma_{\mathrm{LS}} & =\frac{1}{K} \mathrm{E}\left[\left|\boldsymbol{F}\left(\boldsymbol{A}_{\mathrm{I}}^{\mathrm{H}} \boldsymbol{A}_{\mathrm{I}}\right)^{-1} \boldsymbol{A}_{\mathrm{I}}^{\mathrm{H}} \boldsymbol{W}\right|^{2}\right] \\
& =\frac{1}{K} \mathrm{E}\left[\left|\boldsymbol{F} \boldsymbol{A}_{\mathrm{I}}^{+} \boldsymbol{W}\right|^{2}\right] \\
& =\frac{1}{K} \operatorname{tr}\left\{\mathrm{E}\left[\boldsymbol{F} \boldsymbol{A}_{\mathrm{I}}^{+} \boldsymbol{W} \boldsymbol{W}^{\mathrm{H}}\left(\boldsymbol{A}_{\mathrm{I}}^{+}\right)^{\mathrm{H}} \boldsymbol{F}^{\mathrm{H}}\right]\right\} \\
& =\frac{\sigma^{2}}{K} \operatorname{tr}\left\{\boldsymbol{F} \boldsymbol{A}_{\mathrm{I}}^{+}\left(\boldsymbol{A}_{\mathrm{I}}^{+}\right)^{\mathrm{H}} \boldsymbol{F}^{\mathrm{H}}\right\} \\
& =\frac{\sigma^{2} L}{N_{p}}
\end{aligned}
$$

The normalized mean square error (NMSE) of the self-interference channel estimate in digital domain is defined as

$$
\gamma_{\mathrm{LS}}^{\prime} \triangleq \frac{\gamma_{\mathrm{LS}}}{\mathrm{E}\left[\left|H_{\mathrm{I}}(k)\right|^{2}\right]}
$$

The power of the self-interference in digital domain is given by $P_{\mathrm{I}}$, i.e., $P_{\mathrm{I}}=\mathrm{E}\left[\left|H_{\mathrm{I}}(k) X_{\mathrm{I}}(k)\right|^{2}\right]=\sum_{k=0}^{L-1} \sigma_{k}^{2}$. Then, the self-interference to noise ratio is given by INR, i.e., INR $=\frac{P_{\mathrm{I}}}{\sigma^{2}}$,from (12), we obtain

$$
\gamma_{\mathrm{LS}}^{\prime} \triangleq \frac{\sigma^{2} L / N_{p}}{\sum_{k=0}^{L-1} \sigma_{k}^{2}}=\frac{L}{N_{p} \mathrm{INR}}
$$

The digital self-interference cancellation capability is defined as

$$
\begin{aligned}
G_{\mathrm{LS}} & \triangleq 10 \log _{10}\left(\frac{P_{\mathrm{I}}+\sigma^{2}}{P_{\mathrm{dr}}+\sigma^{2}}\right) \\
& =10 \log _{10}\left(\frac{\mathrm{E}\left[\left|H_{\mathrm{I}}(k) X_{\mathrm{I}}(k)\right|^{2}\right]+\sigma^{2}}{\left.\left.\mathrm{E}||\left(H_{\mathrm{I}}(k)-\hat{H}_{\mathrm{I}}(k)\right) X_{\mathrm{I}}(k)\right|^{2}\right]+\sigma^{2}}\right) \\
& =10 \log _{10}\left(\frac{\mathrm{INR}+1}{\gamma_{\mathrm{LS}}^{\prime} \mathrm{INR}+1}\right)
\end{aligned}
$$


Substituting (13) into(14) yields

$$
G_{\mathrm{LS}}=10 \log _{10}\left(\frac{\mathrm{INR}+1}{L / N_{p}+1}\right)
$$

From (15), it is clear that the amount of digital self-interference cancellation increase as the self-interference received power increases. In order to analyze the relation between the increase in the amount of the digital self-interference cancellation and the increase in the self-interference received power,we assume that $\mu=10 \log _{10}$ (INR), then $\mathrm{INR}=10^{\mu / 10}$, and (15) can be expressed as

$$
G_{\mathrm{LS}}=10 \log _{10}\left(\frac{10^{\mu / 10}+1}{L / N_{p}+1}\right)
$$

From(16), the first derivative of the digital self-interference cancellation capability $G_{\mathrm{LS}}$ versus the received self-interference power $\mu$ can be found to be

$$
\eta \triangleq \frac{d G_{\mathrm{LS}}}{d \mu}=\frac{10^{\mu / 10}}{10^{\mu / 10}+1}=\frac{\mathrm{INR}}{\mathrm{INR}+1}
$$

From (17), it should be noted that

i) $\eta<1$, that indicates the decrease in the amount of digital self-interference cancellation is always less than the increase in the self-interference received power in digital domain. If the amount of RF self-interference cancellation increases, the self-interference received power in digital domain will decrease when the self-interference received power at RF front-end is fixed. The decrease in the self-interference received power in digital domain will result in an increase in the error of the self-interference channel estimate in the digital domain and the decrease in the digital self-interference cancellation capability. But the decrease in the amount of digital self-interference cancellation is less than the increase in RF self-interference cancellation. It is clear that the RF self-interference cancellation should be made as much as possible to maximize the total self-interference cancellation capability for FD systems.

ii) $\eta$ will approach 1 as INR increases. That indicates the increase in the amount of digital self-interference cancellation is nearly the same as the increase in the amount of the self-interference received power in digital domain when the latter is large. Applying digital cancellation after RF cancellation is more effective when RF cancellation delivers poor suppression that is consistent with the experimental results in [8].

\section{Numerical and Simulation Results}

In this section, simulations are performed in the matlab simulation software to illustrate the results. We assume that the number of subcarrier is 2048, i.e., $K=2048$. There is a cyclic prefix of 144 samples. The near-end self-interference pilot tones are set to be equipowered and equispaced. i.e., $i_{m}-i_{m-1}=K / N_{p} . N_{p}$ is the number of pilots. The channel has Rayleigh distribution with an exponential power delay profile, i.e., $\sigma_{k}^{2}=\exp (-k / 10), k=0,1, \cdots, L-1$ and $L=40$. 


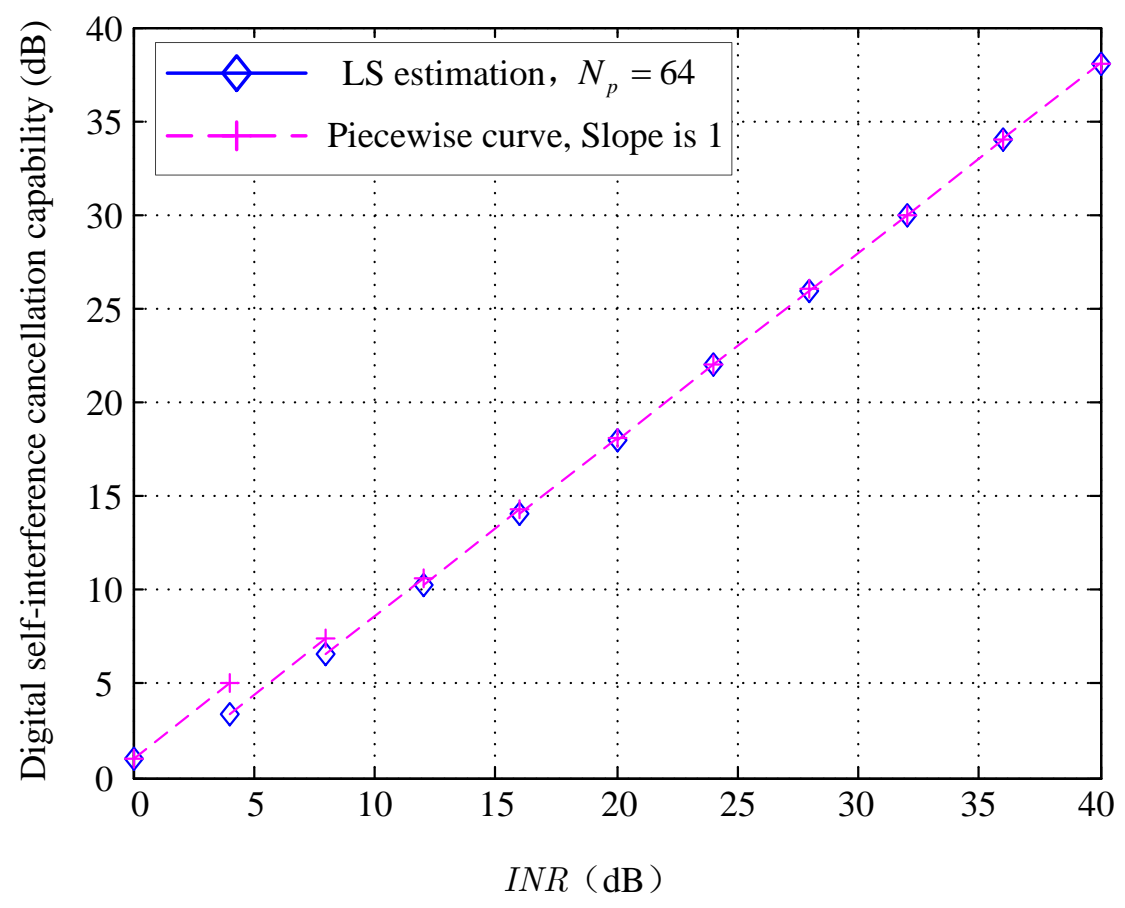

Fig.2. Digital self-interference cancellation capability under different INR

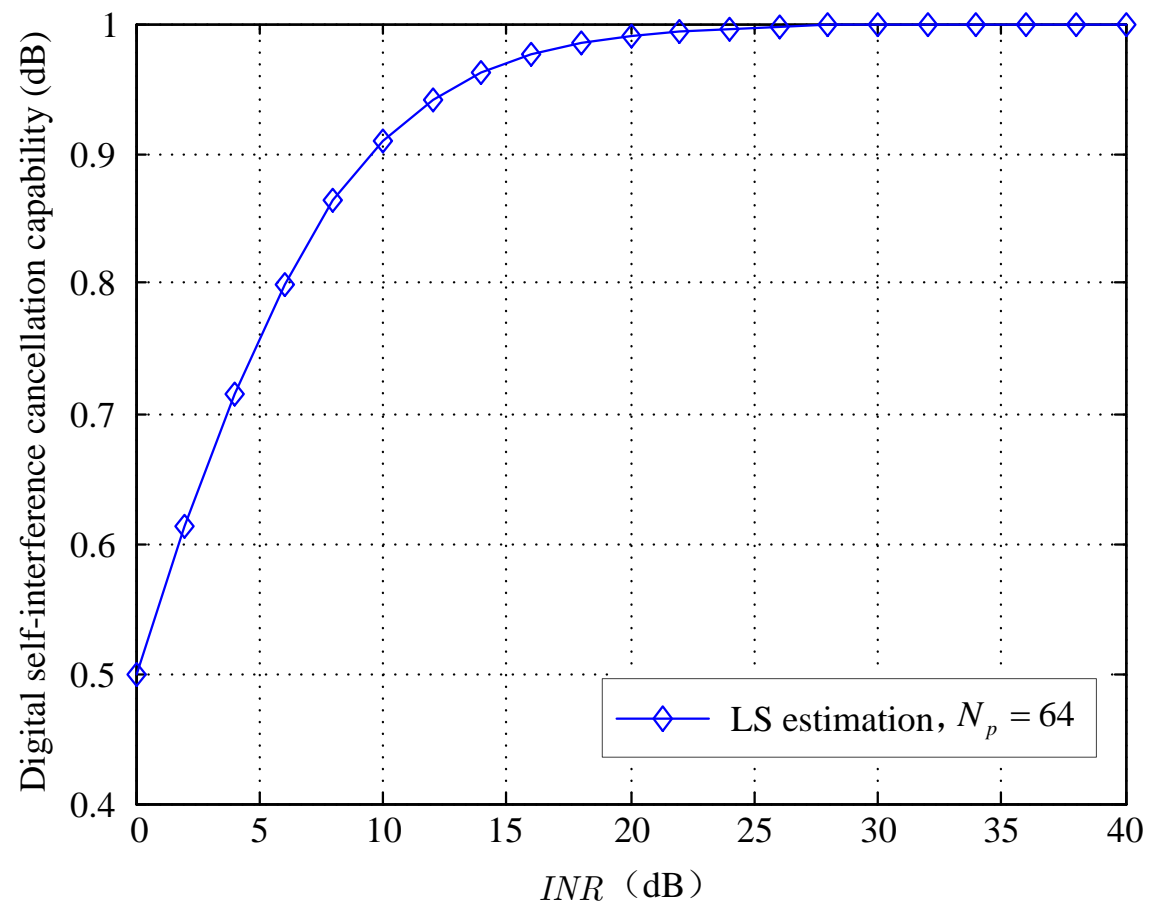

Fig.3. The rate of change of the digital self-interference cancellation capability with respect to the self-interference received power in digital domain

Fig. 2 shows the performance of digital self-interference cancellation capability under different INR. It can be seen that the digital self-interference cancellation capability increases with the increase of the self-interference received power in digital domain. The increase in the amount of digital self-interference cancellation is less than the increase in the self-interference received power in digital domain when INR in digital domain is under $20 \mathrm{~dB}$. The increase in the amount of digital self-interference cancellation is nearly the same as the increase in the self-interference received power in 
digital domain when INR in digital domain is above $20 \mathrm{~dB}$.

Fig.3 illustrate the slope of the curve shown in Fig.2. It shows the rate of change of the digital self-interference cancellation capability with respect to the received power in digital domain. It can be seen that the rate of change of the digital self-interference cancellation capability with respect to the received power in digital domain is always less than one, i.e., $\eta<1$, which is consistent with the theoretical analysis. It is shown that the increase in the amount of RF self-interference cancellation is always more than the decrease in the amount of digital self-interference cancellation. In practice we should let the RF self-interference cancellation be made as much as possible to maximize the total self-interference cancellation capability for FD systems. Fig.3 shows $\eta$ will be close to 1 when $I N R$ in digital domain is above $20 \mathrm{~dB}$, which indicates the increase in the amount of RF self-interference cancellation is nearly equal to the decrease in the amount of digital self-interference cancellation when the amount of RF self-interference cancellation is small. In this case applying digital cancellation after RF cancellation is more effective. From Fig.3, we can see that $\eta$ will decrease rapidly when $I N R$ in digital domain is under $20 \mathrm{~dB}$, which indicates the performance of digital self-interference cancellation will degrade when RF cancellation achieves large suppression.

\section{Conclusion}

According to the view of the information processing inequality of information theory, it is better to solve the self-interference problem at the first step, i.e., in RF domain. We should try to avoid solving the self-interference problem at the second step, i.e., in digital domain. This conclusion has been verified by this paper as the increase in the amount of RF cancellation is always more than the decrease in the digital self-interference cancellation capability. However, due to engineering constraints, the RF self-interference cancellation capability is always limited and the digital self-interference cancellation is needed to reduce the residual self-interference. In this case, the balance between RF and digital self-interference cancellation should be considered. The analysis and simulation show that the amount of RF self-interference cancellation should not be too large to guarantee the effectiveness of the digital self-interference cancellation. Otherwise there will be a decrease in the effectiveness of the digital self-interference cancellation, which results in a decrease in the total amount of self-interference cancellation.

\section{Acknowledgement}

This research was financially supported by the National Natural Science Foundation of China (61531009, 61501093, 61271164, 61471108, 61201266) and the National Major Project of China (2014ZX03003001-002).

\section{References}

[1] GUO Tianwen and WANG Baoyun. Joint transceiver beamforming design for end-to-end optimization in full-duplex MIMO relay system with self-interference[J]. IEEE Communications Letters, 2016, 20(9): 1733-1736. 
[2] MOHAMMADI M, CHALISE B K, SURAWEERA H A, et al. Throughput analysis and optimization of wireless-powered multiple antenna full-duplex relay systems[J]. IEEE Transactions on Communications, 2016, 64(4): 1769-1785.

[3] MOKHTAR M, DHAHIR N A, and HAMILA R. OFDM full-duplex DF relaying under I/Q imbalance and loopback self-interference[J]. IEEE Transactions on Vehicular Technology, 2016, 65(8): 6737-6741.

[4] ZHANG Zhongshan, CHAI Xiaomeng, LONG Keping, et al. Full duplex techniques for $5 \mathrm{G}$ networks: self-interference cancellation, protocol design, and relay selection[J]. IEEE Communications Magazine, 2015, 53(5): 128-137.

[5] KORPI D, RIIHONENY T, and VALKAMA M. Achievable rate regions and self-interference channel estimation in hybrid full-duplex/half-duplex radio links[C]. Proceedings of 2015 49th Annual Conference on Information Sciences and Systems, Baltimore, USA, 2015: 1-6.

[6] TAGHIZADEH $\mathrm{O}$ and MATHAR R. Interference mitigation via power optimization schemes for full-duplex networking[C]. Proceedings of 2015 19th International ITG Workshop on Smart Antennas, Ilmenau, Germany, 2015: 1-7.

[7] MASOUDI A and NGOC T L. Channel estimation and self-interference cancellation in full-duplex communication systems[J]. IEEE Transactions on Vehicular Technology, 2016, PP(99):1-1.

[8] AHMED E and ELTAWIL A M. All-digital self-interference cancellation technique for full-duplex systems[J]. IEEE Transactions on Wireless Communications, 2015, 14(7): 3519-3532.

[9] LI S H and MURCH R D. An investigation into baseband techniques for single-channel full-duplex wireless communication systems[J]. IEEE Transactions on Wireless Communications, 2014, 13(9): 4794-4806.

[10]TAPIO V and SONKKI M. Analog and digital self-interference cancellation for full-duplex transceivers[C]. European Wireless 2016, Berlin, Germany, 2016: 1-5.

[11] VERMEULEN T, LIEMPD B V, HERSHBERG B, et al. Real-time RF self-interference cancellation for in-band full duplex[C]. 2015 IEEE International Symposium on Dynamic Spectrum Access Networks, Stockholm, Sweden, 2015: 275-276.

[12]FOROOZANFARD E, FRANEK O, TATOMIRESCU A, et al. Full-duplex MIMO system based on antenna cancellation technique[J]. Electronics Letters, 2014, 50(16): 1116-1117.

[13]MELISSA D, CHRIS D, and ASHUT S. Experiment-driven characterization of full-duplex wireless systems[J]. IEEE Transactions on Wireless Communications, 2012, 11(12):4296-4307.

[14]BARHUMI I, LEUS G, and MOONEN M. Optimal training design for MIMO OFDM systems in mobile wireless channels[J]. IEEE Transactions on Signal Processing, 2003, 51(6): 1615-1624.

[15] MINN H and AL-DHAHIR N. Optimal training signals for MIMO OFDM channel estimation[J]. IEEE Transactions on Wireless Communications, 2006, 5(5): 1158-1168. 
[16]MORELLI M and MENGALI U. A Comparison of pilot-aided channel estimation methods for OFDM systems[J]. IEEE Transactions on Signal Processing, 2001, 49(12): 3065-3073. 